\title{
Oil metal particles Detection Algorithm Based on Wavelet
}

\section{Transform}

\author{
Wei Shang ${ }^{a}$, Yanshan Wang ${ }^{b}$, Meiju Zhang ${ }^{c}$ and Defeng Liu ${ }^{\mathrm{d}}$ \\ AVIC Beijing Changcheng Aeronautic Measurement and Control Technology Research Institute, Beijing, China \\ ashangwei987654@163.com, ${ }^{b}$ wangys@263.net, ${ }^{c}$ zhangmj_bmc@163.com, ${ }^{d}$ liudf@avic-aim.com
}

\begin{abstract}
In order to observe the real-time abrasion status of the aero-engine, we need to monitor the lubrication system online. As the aero-engine operating time and running state changes, the concentration, composition, size and other parameters of the metal debris can show different changes. They can be used as an important indicator to reflect the state of the aero-engine fault. However, due to the influence of electromagnetic, vibration disturbance and random noise signal introduced by the processing unit itself, the metal particles signal tend to comprise noise. Oil metal particles detection algorithm based on wavelet transform, utilizes the optimized localized nature in time domain and frequency domain of wavelet transform and the characteristics of multi-resolution analysis, combined with the signal characteristics in actual aero-engine condition to realize noise reduction and detection, while validating the algorithm using real experimental data. The result shows that noise can be effectively decreased and signal characteristics can be detected correctly.
\end{abstract}

Keywords: Sensor, metal debris, wavelet transform, variance iterative estimation, feature extraction.

\section{Introduction}

With the rapid development of sensor technology, oil metal particle on-line monitoring sensors have been widely used in aviation, marine power equipment and other online monitoring fields [1]. For example, in aero-engine lubrication system, the content of metal particles, as an important indicator, is often used as the basis for judging the status of the engine [2, 3]. Oil metal particles on-line monitoring sensor can not only detect size of metal particles but also distinguish ferromagnetic and non-ferromagnetic metal particles. The parameter of metal particles can also be used to analyze the health state of the aero-engine.

\section{Oil particle detection algorithms}

\subsection{Structure of the oil metal particles on-line monitoring sensor}

Oil metal particles on-line monitoring sensor comprises sensor probe, signal transmission cable and the signal processing unit, the overall connection shown in Figure 1. 


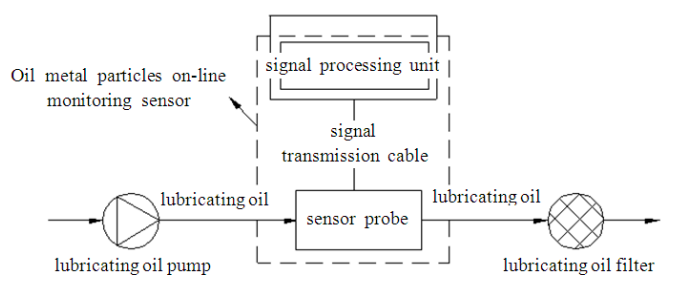

Fig.1. Oil metal particle on-line monitoring sensor overall connection

Sensor probe is installed in the engine oil pipe from filter oil booster to supply pipe, oil can pass through the sensor probe in order to detect metal particles, and signal transmission cable is installed between the sensor probe and the signal processing unit. Sensor probe induce particles signal mainly based on the principle of electromagnetic induction. When a metallic particle passes through the sensor, it generates a full period of sine-like waveform which is considered as particle signature(shown in Figure 6(a) or Figure7(a)). The amplitude of the sine-like waveform signal is proportional to the mass of ferromagnetic and the surface area of non-ferromagnetic, and the phase of them are opposite. Thus, we can obtain the size and the type of the debris by extracting amplitude, phase and frequency information.

However, on-line monitoring sensors are often exposed to vibration interferences during their operations, which will severely reduce the SNR[4] .Thus, it is difficult to estimate the real particle size due to the interference on the sensor. Since metal particle detection is an important aspect of sensor performance, achieving signal from the complex signals is particularly important. This calls for effective de-noising methods to extract the oil debris signature from noisy sensor output data. On the other hand, an ideal de-noising method can completely remove the noise component without any loss in the integrity of the target signal component. However, it is almost impossible to achieve such a goal in real applications. As described above, the oil metal particle monitoring sensors are often exposed to vibration interferences, and the signal is low-intensity and high-frequency wideband, so we can de-noising under the premise of ensuring the energy of signal.

Since oil metal particle on-line monitoring sensor signal is sparse, low frequency, sinusoidal and strong noise, we use a metal particle detection algorithm based on wavelet transform, make full use of the advantages of iterative least squares method and variance estimation algorithm for de-noising, and then complete signal detection according to the true characteristics of signal.

\subsection{Algorithm overall Design}

Depend on the result of signal processing unit design, the sensor signal after AD sampling will produce two route valid signal - the left and the right channel signal, they are respectively sensitive to ferromagnetic particle and non-sensitive ferromagnetic particle. When ferromagnetic particles pass through the sensor, the left and right channel can both generate cosine signal and the amplitude of left channel signal is larger, when the non-ferromagnetic particles pass through the sensor, left and right channel respectively generate cosine and sine signal and the amplitude of right channel signal is larger. The relationship between signal amplitude and phase can be as the basic aspect for signal detection and extraction.

Figure 2 shows the basic flow of data processing. Firstly, left and right channel respectively obtain the original data acquired by $\mathrm{AD}$, then using the least square method to remove baseline wander, eliminating noise by wavelet noise reduction method, using nonlinear energy operator to test ferromagnetic and non-sensitive ferromagnetic particle, finally communicating the particle data up to host computer.

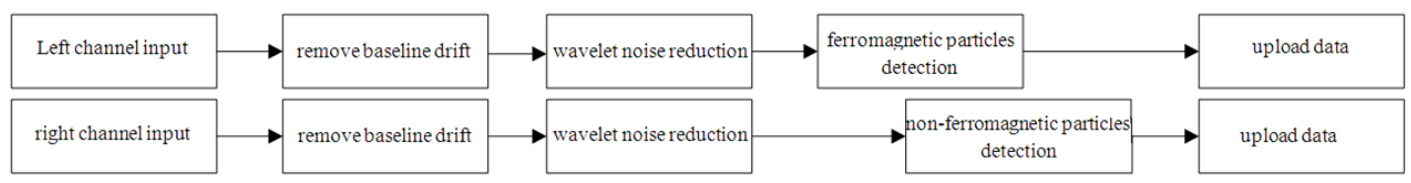

Fig.2. Signal handler Basic Flowchart 


\subsection{Wavelet Analysis}

Figure 5(a) shows a ferromagnetic particle signature collected from oil metal particle on-line monitoring sensor in a vibration-free environment. If a particle signal is too weak, it may be masked by noise and cannot be detected directly. As such, we adopt a wavelet transform approach to detect particle footprint in a noisy environment. Wavelet transform analysis is a very effective tool for time-frequency analysis [5], the most prominent feature is that it has good localization properties and characteristics of multi-resolution analysis in the time and frequency domain. Multi-resolution analysis characteristic signal can be displayed in different levels, and its essence is to restore signal on a range of different levels of space. This restoration can decomposition a variety of signal containing different frequencies into the sub-signal with different frequency bands, which can be effectively used in many signal processing problems.

We can use two sets of filter coefficients $\left\{\mathrm{h}_{\mathrm{n}}\right\}$ and $\left\{\mathrm{g}_{\mathrm{n}}\right\}$ to decompose signal into rough section and detail section, $\left\{\mathrm{h}_{\mathrm{n}}\right\}$ is low-pass filter and $\left\{\mathrm{g}_{\mathrm{n}}\right\}$ is high-pass one. Rough section corresponds to the low-frequency portion of the signal, which represents the main outline of signal, detail part corresponds to the high frequency part of the signal. The two sections are the performance of signal by the wavelet transform in scale 1 . For example, if the signal frequency is $\mathrm{f}_{\mathrm{s}}$, the rough part through $\left\{\mathrm{h}_{\mathrm{n}}\right\}$ will obtains the result which frequency is between 0 and $\mathrm{f}_{\mathrm{s}} / 2$, and detail part will obtains frequency between $\mathrm{f}_{\mathrm{s}} / 2$ and $\mathrm{f}_{\mathrm{s}}$. Then do the same operation on rough section and obtain the result in scale 2 . And so on.

According to the sampling rate of sensor, the decomposition level of oil metal particle on-line monitoring sensor is 7 , and according to the frequency range of signal, making the first five layers of detail coefficients set to 0 in order to remove the high frequency components.

Wavelet de-noising is based on Wavelet transform and it is frequently be used because of its outstanding advantages. Firstly, the original signal is decomposed into multiple scales by using wavelet transform, and then depends on different nature in wavelet coefficients of signal and noise, we can use corresponding roles in signal processing. The essence here is to reduce the coefficient generated by noise, as well as maximizing retaining real signal coefficients, and finally reconstruct the original signal based on wavelet coefficients. The result of the processing of noisy wavelet coefficients greatly affects the signal noise reduction effect, so it is an important part of the noise reduction process. This is described in detail in 2.5 .

\subsection{Remove the trend component}

From above, the signal always includes low frequency trend component due to acquisition error, vibration interferences and other reasons. In normal circumstance, this trend component is usually negligible. However, when dealing with wavelet processing, due to the operational characteristics, low-frequency component is often amplified. It always causes a significant shift so we need take appropriate methods to remove these drift components.

The classical de-trending process such as least-squares scheme can often removes the trend effect by finding the best-fit line and then subtracts it from the original signal. Here, we use this method to remove the trend component. We assume a polynomial trend item and use least squares equation theory to make formula, then take matrix method to solve out the matrix coefficients and obtain trend term, finally original signal can be derived by subtracting the trend term useful signal[6].

Assuming the input data is $X_{\text {in }}[N]$, the amount of data is $N$, use $U_{i}$ to fit trend term

$$
\mathrm{U}_{\mathrm{i}}=\sum_{\mathrm{i}=0}^{\mathrm{N}-1} \mathrm{~b}_{\mathrm{k}} \mathrm{X}_{\mathrm{in}}{ }^{\mathrm{k}}(\mathrm{i}) \text {. }
$$

Where is the polynomial coefficients. According to the principle of least squares error, the error between estimated value and real value is

$$
\mathrm{E}=\sum_{\mathrm{i}=1}^{\mathrm{N}}\left(\mathrm{X}_{\mathrm{in}}(\mathrm{i})-\mathrm{U}_{\mathrm{i}}\right)^{2}
$$

We solve out the minimum error in accordance with the least squares method and solve its partial derivative, then make it zero, sorting out the corresponding equation, using the matrix method for solving polynomial coefficients. In 
the sensor system described in the present, using a polynomial, obtained trend coefficient after solving, then coming to the mathematical expression

$$
\begin{aligned}
& \mathrm{Y}_{\text {out }}(\mathrm{i})=\mathrm{X}_{\text {in }}(\mathrm{i})-\left(\mathrm{a}+\mathrm{b} *\left(\mathrm{i}-\frac{N}{2}\right)\right) . \\
& \mathrm{a}=\frac{\sum_{0}^{N-1} \mathrm{X}_{\mathrm{in}}(\mathrm{i})}{N} . \\
& \quad \mathrm{b}=\frac{\sum_{0}^{N-1} \mathrm{X}_{\mathrm{in}}(\mathrm{i})(\mathrm{i}-\mathrm{a})}{\sum_{0}^{N-1}(\mathrm{i}-\mathrm{a})(\mathrm{i}-\mathrm{a})} .
\end{aligned}
$$

$\mathrm{Y}_{\text {out }}(\mathrm{i})$ is the data item after de-trending.

\subsection{Wavelet domain coefficient adjustment}

The key of wavelet filtering is removing noise component in frequency domain. In the literature, some de-noising methods mainly focus on maintaining the integrity of the target signal component and remove only part of the noise component. These methods are called under-de-noising approaches which may lead to false alarm due to certain spurious noise components such as vibration remaining in the partially denoised data. On the contrary, another kind of methods primarily concentrates on the removal of noise component with less regard to the target signal integrity. In this case, part of the signal component could also be removed, leading to distortion or even loss of the particle signature. Considering of the characters of sensor, we point out a noise variance iterative estimation method which is carried out to removing large deviation from the mean weight, thus gathering signal energy.

\subsubsection{Iterative noise variance estimation}

Variance represents the deviant degree to average value, the part which has larger deviation is considered correlation-weak to valid signal and is not enough to reflect the signal characteristics. Here point out variance iterative estimation method, do the nonzero iterate calculation on wavelet decomposition coefficients, thus can be more accurate to estimate the noise variance. Here we can use threshold to measure the extent.

Firstly over all $\mathrm{N}$ wavelet coefficients $\mathrm{s}(\mathrm{n})$ at layer $\mathrm{j}(\mathrm{j}=6$ and 7)and rough wavelet coefficients separately with the M-length window and with $50 \%$ data overlap, then calculate all window variance. For example, in the detection system, $\mathrm{N}$ takes 2048, M takes 128, so the data is divided into 32 segments, each of 128 data, then calculate each variance, select the minimum $\mathrm{v}_{\min }$, using the following formula to calculation threshold

$$
\mathrm{T}=\sqrt{2 * \mathrm{v}_{\min } * \ln (\mathrm{N})}
$$

Then maintain the data which is between $-\mathrm{T}$ and $\mathrm{T}$, thus constitute a new array and then do the same processing as described above, iterative 3 times, finally obtain the variance of the data retention.

The amplitude of valid signal is relatively concentrated through wavelet transform, so after removing the data outside the threshold, it can effectively remove large deviation from the overall level of the noise signal, the energy will be focused on preserving the data valid signal in order to achieve noise reduction purpose.

\subsubsection{The improve coefficient adjustment method}

There are three major wavelet signal extraction methods that are respectively based on modulus maxima, adjacent scale correlation and threshold shrinkage principles. Since the sensor output data is a mixture of debris signature, white noise and correlated vibration interference, it is very difficult to directly use modulus maxima or correlation-based methods. The threshold shrinkage in the wavelet domain is the most widely used due to its simplicity and effectiveness. There are two basic threshold shrinkage methods. There are hard threshold shrinkage and soft threshold shrinkage. Hard threshold adjustment can achieve a smaller mean square error after signal de-noising, but it has breakpoints, which leads to the shock point mutation after signal de-noising, when there are higher noise level, this phenomenon is particularly obvious. Soft threshold adjustment can achieve greater SNR after signal de-noising, but the wavelet coefficient after Soft threshold adjustment existent constant deviation, which will directly affect the degree of approximation of the reconstructed signal and the real signal, and it can bring inevitable errors to reconstructed signal.

In view of the advantages and disadvantages of threshold processing, here we use an improved threshold processing method. If $\operatorname{swad}(\mathrm{i})>T$, then 


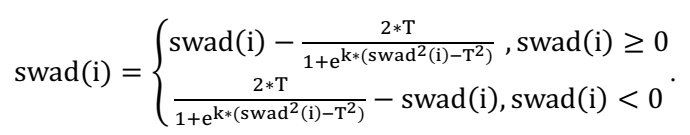

Else $\operatorname{swad}(\mathrm{i})=0 . \mathrm{T}$ is the result in $2.5 .1, \mathrm{k}=1, \mathrm{e}=2.718$, $\operatorname{swad}(\mathrm{i})$ represents detail coefficients and rough wavelet coefficients at layer 6 and 7. This method can effectively improve the breakpoint effects which is induced by hard threshold adjustment method, at the same time, respect to the soft threshold adjustment, it can ensure better continuity and higher accuracy, thus can achieve better filtering purpose.

\subsection{Particle detector}

Here, particle detection method is based on the signal characteristics of oil particles, using nonlinear energy operator which can simultaneously reflect the instantaneous frequency and amplitude information of the signal. Output is proportional to the product of the amplitude and frequency of input signal. For signal sequence $\mathrm{X}$ (i)

$$
\varphi(\mathrm{X}(\mathrm{i}))=\mathrm{X}(\mathrm{i}) * \mathrm{X}(\mathrm{i})-\mathrm{X}(\mathrm{i}-1) * \mathrm{X}(\mathrm{i}+1) .
$$

After nonlinear energy operator processing, signal output is larger at abrasive location, here we can set the threshold $\mathrm{T}$ to initially screened abrasive location. We get $\mathrm{T}$ according to the following formula

$$
\mathrm{T}=\mathrm{C} * \frac{1}{\mathrm{~N}} * \sum_{\mathrm{n}=1}^{\mathrm{N}} \varphi(\mathrm{X}(\mathrm{n})) \text {. }
$$

Here, $\mathrm{C}$ is the empirical constant, according to a large number of statistical results in this project, takes 2 . Select the part which signal energy is above $\mathrm{T}$ to initially regard as the effective particle signal.

Then do further choose to the position which is obtained by non-linear energy operator, It includes: abrasive speed, symmetry and amplitude. Here the left channel is sensitive for ferromagnetic and it shows cosine phase which valley precedes peak in the post; the right channel is sensitive for non-ferromagnetic and it shows cosine phase which peak precedes valley in the post. By determining the particle morphology and symmetry to eliminate abnormal signal, then set the appropriate threshold based on the nature and magnitude, excluding the impact of mutations and small-amplitude interference signal.

\section{Results and Discussion}

In this section, we use oil particles online monitoring sensor signal detection algorithms mentioned above to test the actual data in a lab environment. The oil particles online monitoring sensor is shown in Figure 3.

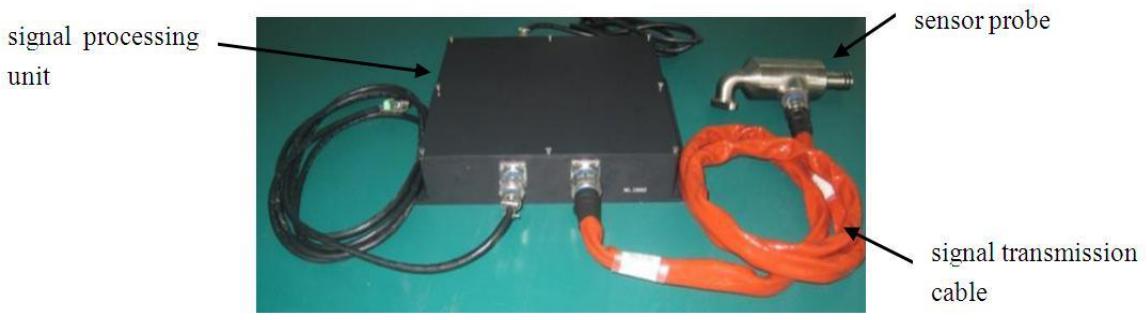

Fig.3. Oil particles online monitoring sensor

Take an $110 \mu \mathrm{m}$ ferromagnetic metal particle and a $508 \mu \mathrm{m}$ non-ferromagnetic metal particle which were embedded in a plastic sleeve (shows in Figure 4). In the test, in accordance with the requirements of sensor, respectively pump plastic sleeve through the sensor with the speed within $1 \sim 4 \mathrm{~m} / \mathrm{s}$ to ensure effective through of metal particles. 


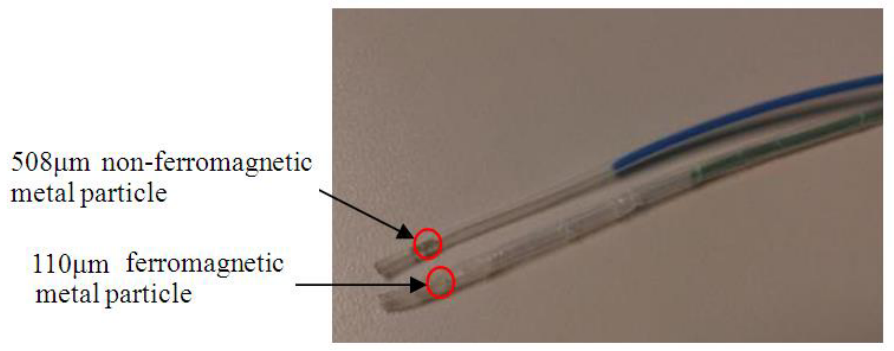

Fig.4. $110 \mu \mathrm{m}$ ferromagnetic metal particle and $508 \mu \mathrm{m}$ non-ferromagnetic metal particle

Connect the sensor system and give power, open PC software for testing. Smoke particles and observe original sensor signal and signal after processing, the result shows in Figure 5-6:

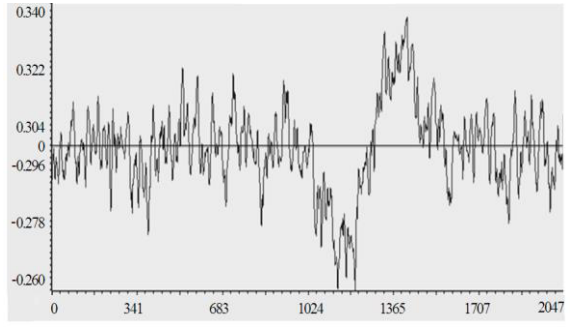

(a)

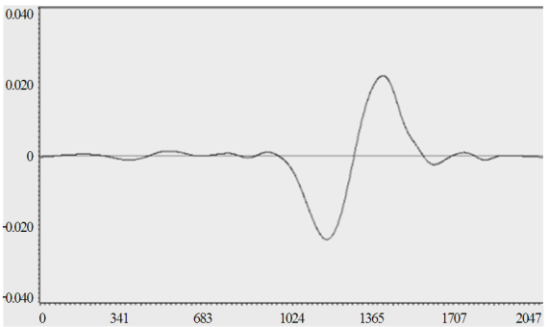

(b)

Fig.5. $110 \mu \mathrm{m}$ Ferromagnetic metal particle

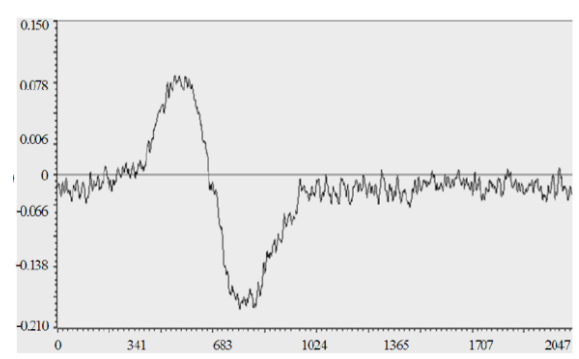

(a)

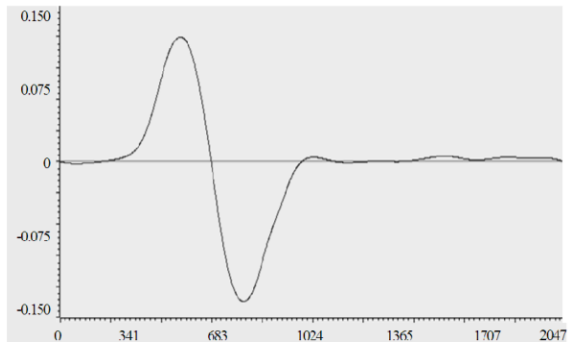

(b)

Fig.6. 508 $\mu \mathrm{m}$ Non-ferromagnetic metal particle

In Figure 5-6, (a) shows the raw data measured from the sensor, and (b) shows the data after disposing. As shown, the graphic above displays the original signal of metal particles, the graphic below shows the filtered signals after filtering, the indmax and indmin show the peak and valley position of waveform. As can be seen, the filtered signal has dropped baseline drift and has no noise. It can fully reproduce the original signal significantly, enhance signal to noise ratio, and can correctly point out the signal peak position.

\section{Conclusion}

A wavelet domain de-noising technique has been proposed to extract high quality debris signature for improving the measurement capability of the oil debris monitoring sensor. Metal particle detection algorithm has fully considered the impacts of both target and unwanted signals, it based on wavelet transform, using the least squares method to remove trend component, effectively avoiding to enlarge the low frequency component when doing wavelet transform. Other advantages of this method include the iterative variance estimation can effectively remove the remote components from 
the mean level of the signal. This method has been evaluated using sensor data, the results show the algorithm can effectively improve the oil debris monitoring accuracy in real applications where vibration interferences are inevitable.

So far, the application of the particle detection algorithm has successfully passed the test of the engine, and the present system has completed XX-engine two phases test and delivery.

\section{References}

1. J.L. Miller, D. Kitaljevch, In-line oil debris monitor for aircraft engine condition assessment, IEEE Aerospace Conference Proceedings, 6(2000)

2. Q.B. He, X.X. Wang, Q. Zhou, Vibration sensor data denoising using a time-frequency manifold for machinery fault diagnosis, 14(2014)

3. J. Wu, T.G. Liu, X. Tang, Qualitative ferrographic analysis method by quantitative parameter of wear debris characteristics, Industrial Lubrication and Tribology, 64, 6(2012)

4. R.W. Kempster, D.B. George, Method and apparatus for detecting particles in a fluid having coils isolated from external vibrations, United States Patent, 5(1995)

5. Y. Zhang, S.R. Lee, H. Zhao, B. Wang, B. Liu, Research on wavelet analysis in fault signal processing. International Conference on Signal Processing proceedings, 1(2004)

6. S.C. Chan, H.C. Wu, K.M. Tsui, A new method for preliminary identification of gene regulatory networks from gene microarray cancer data using ridge partial least aquares with recursive feature elimination and novel brier and occurrence probability measures, IEEE Transactions on System, Man and Cybernetics, 42, 6( 2012) 